\title{
Growth phase-dependent control of transcription start site selection and gene expression by nanoRNAs
}

\author{
Irina O. Vvedenskaya, ${ }^{1,2}$ Josh S. Sharp, ${ }^{3,6}$ Seth R. Goldman, ${ }^{1,2,6}$ Pinal N. Kanabar, ${ }^{1}$ Jonathan Livny, ${ }^{4,5}$ \\ Simon L. Dove, ${ }^{3,7}$ and Bryce E. Nickels ${ }^{1,2,7}$ \\ ${ }^{1}$ Waksman Institute, Rutgers University, Piscataway, New Jersey 08854, USA; ${ }^{2}$ Department of Genetics, Rutgers University, \\ Piscataway, New Jersey 08854, USA; ${ }^{3}$ Division of Infectious Diseases, Boston Children's Hospital, Harvard Medical School, \\ Boston, Massachusetts 02115, USA; ${ }^{4}$ The Broad Institute of Massachusetts Institute of Technology and Harvard, Cambridge, \\ Massachusetts 02142, USA; ${ }^{5}$ Channing Laboratory, Brigham and Women's Hospital, Harvard Medical School, Boston, \\ Massachusetts 02115, USA
}

Prokaryotic and eukaryotic RNA polymerases can use 2- to 4-nt RNAs, "nanoRNAs," to prime transcription initiation in vitro. It has been proposed that nanoRNA-mediated priming of transcription can likewise occur under physiological conditions in vivo and influence transcription start site selection and gene expression. However, no direct evidence of such regulation has been presented. Here we demonstrate in Escherichia coli that nanoRNAs prime transcription in a growth phase-dependent manner, resulting in alterations in transcription start site selection and changes in gene expression. We further define a sequence element that determines, in part, whether a promoter will be targeted by nanoRNA-mediated priming. By establishing that a significant fraction of transcription initiation is primed in living cells, our findings contradict the conventional model that all cellular transcription is initiated using nucleoside triphosphates (NTPs) only. In addition, our findings identify nanoRNAs as a previously undocumented class of regulatory small RNAs that function by being directly incorporated into a target transcript.

[Keywords: RNA polymerase; small RNAs; transcription initiation; gene regulation]

Supplemental material is available for this article.

Received March 23, 2012; revised version accepted May 21, 2012.

The term "nanoRNA" refers to RNA transcripts 2 to $\sim 4$ nt in length (Mechold et al. 2007). NanoRNAs can, in principle, be generated via a number of distinct cellular processes, including RNA degradation and abortive transcription initiation (for review, see Nickels and Dove 2011). Nevertheless, whether nanoRNAs can accumulate to sufficient concentrations to exert functional roles under physiological conditions has not been established.

Prior studies of mutant cells in which nanoRNA concentrations were elevated suggest a potential functional role for nanoRNAs in the regulation of transcription start site selection and gene expression (Goldman et al. 2011). In particular, studies of RNA metabolism in bacteria have identified specialized enzymes, "nanoRNases," which are responsible for the degradation of nanoRNAs (Datta and Niyogi 1975; Ghosh and Deutscher 1999; Mechold et al. 2007; Fang et al. 2009; Liu et al. 2012). Thus, inactivation of an endogenous nanoRNase in Pseudomonas aerugi-

\footnotetext{
${ }^{6}$ These authors contributed equally to this work.

${ }^{7}$ Corresponding authors

E-mail bnickels@waksman.rutgers.edu

E-mail simon.dove@childrens.harvard.edu

Article is online at http://www.genesdev.org/cgi/doi/10.1101/gad.192732.112.
}

nosa causes nanoRNA concentrations to increase and leads to widespread use of nanoRNAs by RNA polymerase as primers for transcription initiation (Goldman et al. 2011). Under these artificial conditions, nanoRNAmediated priming causes alterations in transcription start site selection at the majority of cellular promoters and widespread changes in gene expression. These findings raise the possibility that nanoRNA-mediated priming of transcription initiation may contribute to transcription start site selection and gene expression during physiological growth conditions.

Here we investigated whether nanoRNA-mediated priming occurs during physiological growth conditions in Escherichia coli. We found that nanoRNA-mediated priming occurs in a growth phase-dependent manner and controls transcription start site selection and gene expression. In particular, we demonstrate that growth phasedependent changes in transcription start site selection occur as a direct result of nanoRNA-mediated priming in the context of at least five promoters. We further show that growth phase-dependent nanoRNA-mediated priming leads to an increase in the expression of at least two of the genes associated with these promoters, establishing 
that nanoRNAs play a regulatory role. We define a sequence element, termed the "nanoRNA response element," which determines, in part, whether a promoter will be susceptible to nanoRNA-mediated start site control. In addition, we present evidence that transcripts generated by nanoRNA-mediated priming carry a $5^{\prime}$ hydroxyl, suggesting that the nanoRNAs that prime transcription themselves carry a $5^{\prime}$ hydroxyl. Our findings indicate that nanoRNAs represent a previously undocumented class of regulatory small RNAs that can accumulate to sufficient concentrations to exert functional roles under physiological conditions. In addition, by demonstrating that a significant fraction of transcription initiation in living cells can be primed, our findings contradict the conventional model that all cellular transcription is initiated using nucleoside triphosphates (NTPs) only (i.e., de novo).

\section{Results}

Experimental strategy: determine the effect of ectopic production of a nanoRNase on transcription start site selection

We sought to determine whether nanoRNA-mediated priming of transcription initiation occurs during physiological growth conditions in E. coli. Prior studies had shown that increasing the concentrations of nanoRNAs in P. aeruginosa by inactivating an endogenous nanoRNase leads to widespread alterations in transcription start site selection (Goldman et al. 2011). Therefore, we reasoned that if the concentration of nanoRNAs under physiological growth conditions was sufficient to prime transcription initiation, we should observe an effect on transcription start site selection if nanoRNA concentrations were reduced by overproduction of a nanoRNase (Fig. 1). Accordingly, we determined whether overproducing the nanoRNase that is native to E. coli, Oligoribonuclease (Orn) (Ghosh and Deutscher 1999|, or ectopically producing a heterologous nanoRNase, NrnB from Bacillus subtilis (Fang et al. 2009), altered transcription start site selection. As a control, we also determined the effect of ectopically producing a catalytically inactive version of $\mathrm{NrnB}\left(\mathrm{NrnB}^{\mathrm{DHH}}\right)$ (Fang et al. 2009).

\section{Ectopic production of a nanoRNase alters transcription start site selection during the stationary phase of growth but not during the exponential phase of growth}

We used high-throughput sequencing to assess transcription start site selection. Prior studies in $P$. aeruginosa revealed that the vast majority of the nanoRNA-mediated priming observed when nanoRNA concentrations were elevated involved nanoRNAs carrying either a $5^{\prime}$ monophosphate or a 5' hydroxyl group (Goldman et al. 2011). Therefore, in parallel, we sequenced the $5^{\prime}$ ends of transcripts carrying a $5^{\prime}$ triphosphate only (which includes those that result from initiation with NTPs) and the $5^{\prime}$ ends of all transcripts regardless of the phosphorylation status of the $5^{\prime}$ end (which includes those that result from priming with a nanoRNA carrying a $5^{\prime}$ monophosphate or a $5^{\prime}$ hydroxyl).

We first analyzed transcripts carrying a $5^{\prime}$ triphosphate to define a set of primary transcription start sites (designated position +1 ) and their associated promoters. Next, we analyzed the $5^{\prime}$ ends of all transcripts regardless of their $5^{\prime}$ phosphorylation status and determined the fraction of transcripts initiated from each template position within a window that spanned from a template position 3 base pairs (bp) upstream of the primary transcription start site (i.e., position -3 ) to a template position $2 \mathrm{bp}$ downstream from the primary transcription start site (i.e., position +3 ). We compared the distribution of transcripts initiated from positions -3 to +3 that was observed in cells containing wild-type concentrations of nanoRNAs with the distribution that was observed in cells in which Orn, $\mathrm{NrnB}$, or $\mathrm{NrnB}^{\mathrm{DHH}}$ was ectopically produced. In vitro studies indicate that 2- to 4-nt nanoRNAs can alter the transcription start site to upstream template positions (i.e., positions $-1,-2$, or -3 ) but not to downstream template positions (i.e., positions +2 or +3 ) (Grachev et al. 1984; Ruetsch and Dennis 1987; Goldman et al. 2011). Therefore, we hypothesized that if the concentrations of nanoRNAs in wild-type E. coli cells were sufficient to prime transcription initiation at certain promoters, we should observe a reduction in transcripts initiating from positions $-1,-2$, or -3 at these promoters in cells in which either Orn or NrnB was ectopically produced (Fig. 1).

We analyzed transcription start sites during the exponential and stationary phases of growth. We calculated the distribution of transcripts initiated from positions -3 to +3 for 270 start sites during exponential phase (Supplemental Table 1) and 225 start sites during stationary phase (Supplemental Table 2). Ectopic production of either Orn or NrnB did not result in a significant reduction in the percentage of transcripts initiating from positions $-1,-2$, or -3 for any of the 270 transcription start sites analyzed during exponential

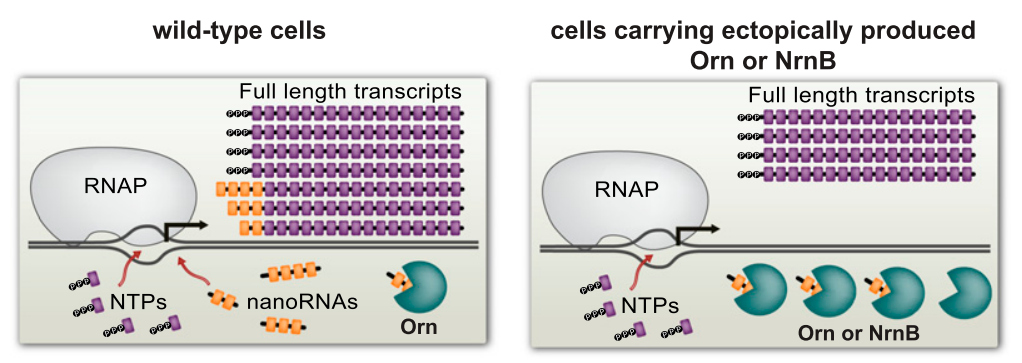

Figure 1. Ectopic production of a nanoRNase (Orn or $\mathrm{NrnB}$ ) prevents RNA polymerase (RNAP) from using nanoRNAs as primers for transcription initiation. 
phase (Fig. 2A; Supplemental Table 1). In contrast, the transcription start sites associated with seven promoters were significantly affected by ectopic production of either Orn or $\mathrm{NrnB}$, but not $\mathrm{NrnB}^{\mathrm{DHH}}$, during stationary phase (Fig. 2B; Supplemental Table 3). On average, for these seven loci, $\sim 50 \%$ of transcripts were initiated from position -1 , while $\sim 50 \%$ of transcripts were initiated from position +1 in cells containing wildtype concentrations of nanoRNAs or in cells in which $\mathrm{NrnB}^{\mathrm{DHH}}$ was ectopically produced (Fig. 2B; Supplemental Table 3). In contrast, in cells in which Orn or NrnB were ectopically produced, only $\sim 15 \%$ of transcripts were initiated from position -1 , while $\sim 85 \%$ of transcripts were initiated from position +1 (Fig. 2B; Supplemental Table 3). Thus, ectopic production of a nanoRNase alters transcription start site selection at a subset of promoters during the stationary phase of growth but not during the exponential phase of growth.

\section{Alterations in transcription start site selection observed upon ectopic production of a nanoRNase are not the result of alterations in NTP concentrations}

It is well established that alterations in the concentrations of NTPs can influence transcription start site selection (Liu et al. 1994; Qi and Turnbough 1995; Tu

\section{A}

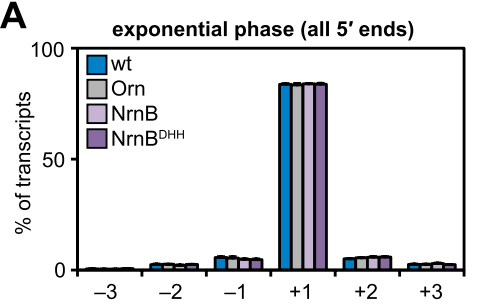

B

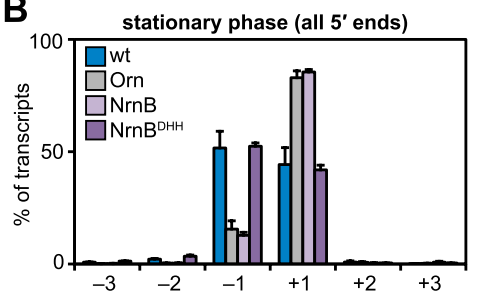

c
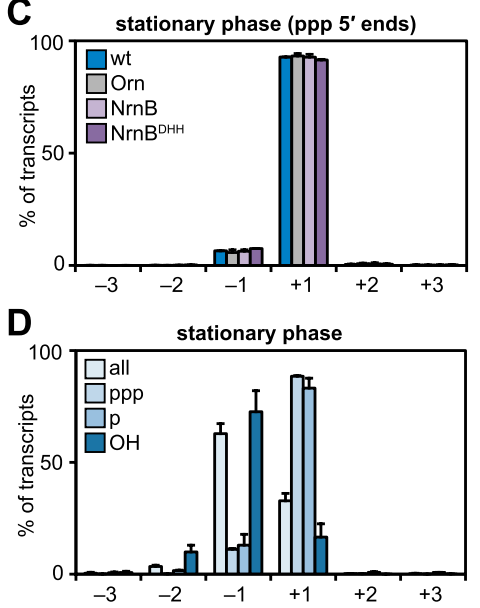

and Turnbough 1997; Meng et al. 2004). If changes in NTP concentrations were responsible for the alterations in transcription start site selection observed during the stationary phase of growth upon ectopic production of a nanoRNase, then we would expect to see start site alterations in both the analysis of all $5^{\prime}$ ends and the analysis of only those transcripts that carry a $5^{\prime}$ triphosphate. However, no alterations in transcription start site selection were observed when only transcripts that carry a 5' triphosphate were analyzed (Fig. 2C; Supplemental Table 3). Thus, the effect of ectopic production of a nanoRNase on transcription start site selection is not a consequence of alterations in the intracellular concentrations of NTPs. We therefore conclude that the alterations in transcription start site selection observed when either Orn or NrnB is ectopically produced during stationary phase are a consequence of a reduction in nanoRNA concentrations that, in turn, causes a reduction in the extent of nanoRNA-mediated priming of transcription initiation.

Transcripts that are sensitive to ectopic production of a nanoRNase during the stationary phase of growth carry a 5' hydroxyl

The data presented in Figure 2, B and C, reveal that transcripts initiating from position -1 of the seven promoters with nanoRNase-sensitive transcription start sites are observed in the analysis of all $5^{\prime}$ ends but not in the analysis of only those transcripts that carry a $5^{\prime}$ triphos-

Figure 2. Effects of ectopic production of a nanoRNase on transcription start site selection. (A) Analysis of transcription start sites during the exponential phase of growth. Average percentage of all transcripts initiated at positions -3 to +3 for the 270 promoters listed in Supplemental Table 1 as determined by high-throughput sequencing. Analysis was performed using RNA transcripts isolated from $E$. coli cells during the exponential phase of growth that harbored an empty plasmid (wt) or a plasmid that specifies production of $E$. coli Orn, B. subtilis $\mathrm{NrnB}$, or $\mathrm{NrnB}^{\mathrm{DHH}}$. Plotted are the mean $+\mathrm{SD}$ derived from two independent measurements. $(B, C)$ Analysis of transcription start sites during the stationary phase of growth. Average percentage of all transcripts $(B)$ or $5^{\prime}$ triphosphate-carrying transcripts $(C)$ initiated at positions -3 to +3 for the seven promoters with start sites that were significantly affected by ectopic production of a nanoRNase (Supplemental Table 3) as determined by highthroughput sequencing. Analysis was performed using RNA transcripts isolated from $E$. coli cells during the stationary phase of growth that harbored an empty plasmid (wt) or a plasmid that specifies production of E. coli Orn, B. subtilis NrnB, or $\mathrm{NrnB}^{\mathrm{DHH}}$. Plotted are the mean $+\mathrm{SD}$ derived from two independent measurements. $(D)$ Average percentage of all transcripts (all), 5' triphosphate-carrying transcripts (ppp), 5' monophosphate-carrying transcripts $(\mathrm{p})$, or $5^{\prime}$ hydroxyl-carrying transcripts $(\mathrm{OH})$ initiated at positions -3 to +3 for the four promoters listed in Supplemental Table 4 as determined by high-throughput sequencing. Analysis was performed using RNA transcripts isolated from $E$. coli cells during the stationary phase of growth that harbored an empty plasmid. Plotted are the mean + SD derived from two independent measurements. 
phate. Thus, transcripts initiating from position -1 of these seven promoters must carry either a $5^{\prime}$ monophosphate or a 5' hydroxyl group. To distinguish between these two possibilities, we used high-throughput sequencing to analyze only those transcripts carrying a $5^{\prime}$ monophosphate or only those transcripts carrying a $5^{\prime}$ hydroxyl that were present in cells carrying wild-type concentrations of nanoRNAs. From this analysis we obtained sufficient sequencing depth to compare the distribution of transcripts carrying a $5^{\prime}$ monophosphate and transcripts carrying a $5^{\prime}$ hydroxyl for four of the seven nanoRNase-sensitive start sites (Fig. 2D; Supplemental Table 4). This analysis revealed that the majority of transcripts initiating from position -1 that are sensitive to ectopic production of either Orn or NrnB carry a 5' hydroxyl group. Therefore, we conclude that the effect of overproducing Orn or ectopically producing NrnB on transcription start site selection during stationary phase is a consequence of a reduction in the concentrations of nanoRNAs carrying a 5' hydroxyl group that, in turn, reduces the extent of nanoRNA-mediated priming.

\section{Growth phase-dependent control of transcription start site selection by nanoRNA-mediated priming}

Five of the seven promoters with nanoRNase-sensitive transcription start sites were represented in the analyses of transcripts isolated during exponential phase and stationary phase (Supplemental Table 5). On average, for these five promoters, $\sim 12 \%$ of transcripts were initiated from position -1 and $\sim 85 \%$ of transcripts were initiated from position +1 during exponential phase, whereas $\sim 60 \%$ of transcripts were initiated from position -1 and $\sim 40 \%$ of transcripts were initiated from position +1 during stationary phase in cells containing wild-type concentrations of nanoRNAs (Fig. 3A; Supplemental Table 5). Furthermore, the distributions of transcription start sites observed during stationary phase in cells in which Orn or NrnB was ectopically produced resembled those observed during exponential phase in cells containing wild-type concentrations of nanoRNAs (Fig. $3 \mathrm{~A})$, suggesting that nanoRNA-mediated priming causes growth phase-dependent changes in start site selection.

To confirm the results of our high-throughput sequencing analyses, we used primer extension to compare the distribution of transcription start sites during exponential phase and stationary phase for the promoters associated with bhs $A$ and $\operatorname{tom} B$, which are the two promoters that were most susceptible to nanoRNAmediated start site control as detected by sequencing. Primer extension analysis of transcripts initiated from plasmid-borne copies of the bhs $A$ and tom $B$ promoters revealed that, for both promoters, essentially all transcripts were initiated from position +1 during exponential phase (Fig. 3B). In contrast, during stationary phase, $>50 \%$ of the transcripts associated with the tom $B$ promoter and nearly all of the transcripts associated with the bhs $A$ promoter were initiated from position -1 (Fig. 3B). Ectopic production of either Orn or NrnB during stationary phase significantly reduced the production

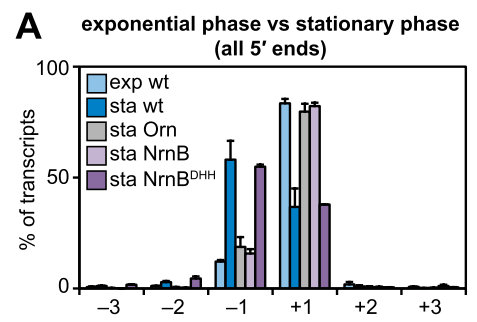

B

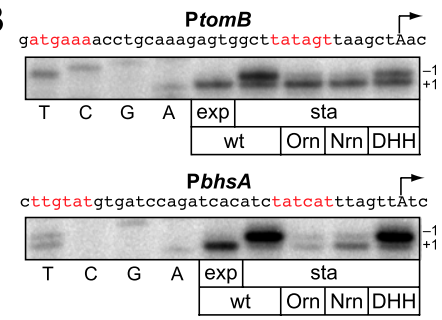

Figure 3. Growth phase-dependent regulation of transcription start site selection by nanoRNA-mediated priming. (A) Average percentage of all transcripts initiated at positions -3 to +3 for the five promoters listed in Supplemental Table 5 as determined by high-throughput sequencing. Analysis was performed using RNA transcripts isolated from E. coli cells during the exponential phase of growth (exp) or the stationary phase of growth (sta) from cells harboring an empty plasmid (wt) or a plasmid that specifies production of $E$. coli Orn, B. subtilis NrnB, or $\mathrm{NrnB}^{\mathrm{DHH}}$. Plotted are the mean $+\mathrm{SD}$ derived from two independent measurements. $(B)$ Primer extension analysis of transcript $5^{\prime}$ ends generated during transcription initiation from plasmid-borne copies of the promoters associated with tomB and $b h s A$. Analysis was performed using RNA transcripts isolated from E. coli cells during the exponential phase of growth (exp) or the stationary phase of growth (sta). Cells harbored a plasmid carrying the indicated promoter along with an empty plasmid $(\mathrm{wt})$ or a plasmid that specifies production of E. coli Orn, B. subtilis $\mathrm{NrnB}$, or $\mathrm{NrnB}^{\mathrm{DHH}}$. Putative -10 and -35 elements of each promoter are highlighted in red. Position +1 is indicated by the arrow.

of transcripts initiating from position -1 of each promoter, whereas ectopic production of $\mathrm{NrnB}^{\mathrm{DHH}}$ did not (Fig. 3B). We conclude, on the basis of the analysis of transcription start sites by high-throughput sequencing (Fig. 3A) and by primer extension (Fig. 3B), that nanoRNAmediated priming regulates transcription start site selection in a growth phase-dependent manner. Our findings indicate that, for at least five promoters, growth phasedependent changes in transcription start site selection occur as a direct result of nanoRNA-mediated priming. Specifically, during stationary phase, nanoRNA-mediated priming leads to the production of transcripts initiated from a template position 1 bp upstream of the transcription start site that is observed during exponential phase.

\section{Growth phase-dependent nanoRNA-mediated priming controls gene expression}

We noticed that ectopic production of either Orn or NrnB reduces the abundance of transcripts initiating from plasmid-borne copies of the tom $B$ and bhs $A$ promoters 
during stationary phase when assessed by primer extension (Fig. 3B). We therefore wished to establish whether ectopic production of a nanoRNase reduces expression of the endogenous tom $B$ gene (which encodes an antitoxin protein) (García-Contreras et al. 2008) and the endogenous bhs $A$ gene (which encodes an outer membrane protein involved in copper permeability, stress resistance, and biofilm formation) (Zhang et al. 2007; Mermod et al. 2012). To do this, we used Northern blotting to compare the abundance of tom $B$ transcripts and $b h s A$ transcripts in cells containing wild-type concentrations of nanoRNAs with the abundance of these transcripts in cells in which NrnB was ectopically produced. Ectopic production of NrnB reduced the abundance of $t o m B$ and $b h s A$ transcripts (approximately fivefold) during stationary phase, but had no effect on the abundance of these transcripts during exponential phase (Fig. 4A). Quantitative real time RT-PCR analysis also demonstrated that ectopic production of a nanoRNase specifically reduced the abundance of bhs $A$ transcripts during stationary phase (Fig. 4B). Thus, ectopic production of a nanoRNase reduces both nanoRNAmediated priming at the tom $B$ and $b h s A$ promoters (Figs. $2,3)$ and expression of these genes during stationary phase (Fig. 4). We conclude that growth phase-dependent nanoRNA-mediated priming regulates expression of the tom $B$ and $b h s A$ genes.
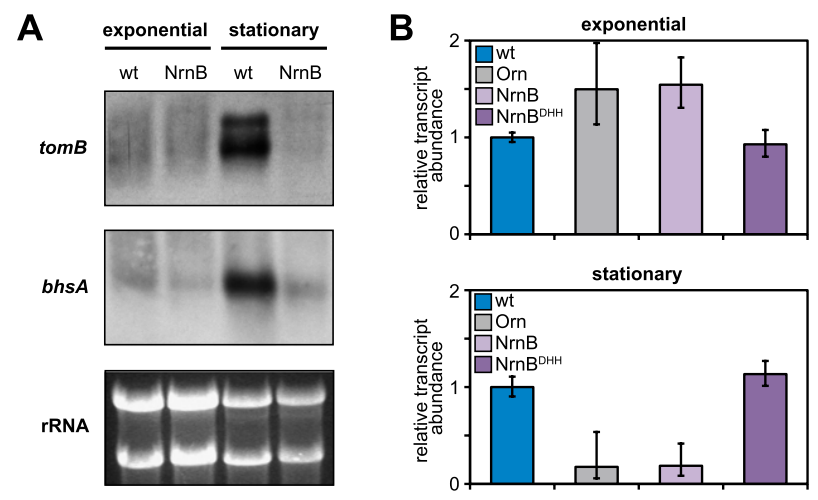

Figure 4. Growth phase-dependent regulation of gene expression by nanoRNA-mediated priming. (A) Northern blot analysis of tomB and bhs $A$ transcripts during the exponential phase of growth or the stationary phase of growth in cells that harbored an empty plasmid (wt) or a plasmid that specifies production of $B$. subtilis NrnB. The bottom panel shows an ethidium bromide-stained gel of the RNA samples used for Northern blotting. The two most prominent bands correspond to the $23 \mathrm{~S}$ and $16 \mathrm{~S}$ rRNAs. (B) Graphs show the abundance of bhsA transcripts relative to the abundance of $r p o D$ transcripts during the exponential phase of growth or the stationary phase of growth in cells that harbored an empty plasmid (wt) or a plasmid that specifies production of E. coli Orn, B. subtilis NrnB, or $\mathrm{NrnB}^{\mathrm{DHH}}$. Relative transcript abundance was measured by quantitative real-time RT-PCR using the comparative $\mathrm{Ct}$ method $(\Delta \Delta \mathrm{Ct})$ (Livak and Schmittgen 2001). Plotted are the means derived from three independent measurements. Error bars represent the relative expression values calculated from plus or minus one SD from the mean $\Delta \Delta \mathrm{Ct}$.
Identification of a sequence element, -1/+1 TA, which determines, in part, whether a promoter will be susceptible to nanoRNA-mediated start site control

Analysis of the sequences of the promoters associated with the seven nanoRNase-sensitive start sites revealed that all of them carried a $\mathrm{T}$ at position -1 and an $\mathrm{A}$ at position +1 (Fig. 5A). Thus, promoters carrying a $-1 /+1$ TA sequence element appear to be particularly sensitive to growth phase-dependent nanoRNA-mediated start site control. To investigate this hypothesis, we compared the distributions of the 44 out of 225 start sites observed during stationary phase that were associated with promoters that carried a $-1 /+1$ TA sequence element with the distributions of the 181 out of 225 start sites associated with promoters that did not carry this sequence element (Fig. 5B; Supplemental Table 2). On average, for the 44 promoters that carried a $-1 /+1 \mathrm{TA}$, $\sim 18 \%$ of transcripts were initiated from position -1 and $\sim 77 \%$ of transcripts were initiated from position +1 in cells containing wild-type concentrations of nanoRNAs (Fig. 5B, top). Ectopic production of either Orn or NrnB (but not $\mathrm{NrnB}^{\mathrm{DHH}}$ ) reduced the percentage of transcripts initiating from position -1 from $\sim 17 \%$ to $\sim 6 \%$ while increasing the percentage of transcripts initiating from position +1 to $\sim 89 \%$ (Fig. $5 \mathrm{~B}$, top). In contrast, on average, for the 181 promoters that did not carry a $-1 /+1 \mathrm{TA}, \sim 6 \%$ of transcripts were initiated from position -1 in cells containing wild-type concentrations of nanoRNAs and $\sim 5 \%$ of transcripts were initiated from position -1 in cells in which either Orn or NrnB were ectopically produced (Fig. 5B, bottom). These data suggest that promoters carrying a $-1 /+1$ TA sequence element are prone to growth phase-dependent nanoRNA-mediated start site control.

As an initial test of the role of the $-1 /+1$ TA sequence element in growth phase-dependent nanoRNA-mediated priming, we determined the effect of mutating this sequence element. To do this, we introduced an $\mathrm{A}+1 \mathrm{G}$ substitution into the plasmid-borne copies of the bhs $A$ and $\operatorname{tom} B$ promoters. We then used primer extension analysis to determine whether ectopic production of $\mathrm{NrnB}$ affected transcription start site selection in the context of the $A+1 G$ derivatives of the bhs $A$ and tom $B$ promoters. Whereas ectopic production of $\mathrm{NrnB}$ altered transcription start site selection during the stationary phase of growth in the context of the wild-type bhs $A$ and tom $B$ promoters (Figs. 3B, 5C), ectopic production of $\mathrm{NrnB}$ had no effect on transcription start site selection in the context of the A+1G promoter derivatives (Fig. 5C). Thus, mutating the $-1 /+1$ TA sequence element in the context of the bhs $A$ and tom $B$ promoters eliminates growth phase-dependent nanoRNA-mediated priming.

We next determined the effect of introducing $-1 /+1$ TA into the context of a heterologous promoter (PlacUV5, a constitutive version of the E. coli lac promoter) (Arditti et al. 1968). As shown in Figure 5D, transcription initiation from a derivative of PlacUV5 carrying a CA at positions $-1 /+1$ began at only position +1 during both exponential phase and stationary phase. In contrast, 
transcription initiation from a derivative of placUV5 carrying a TA at positions $-1 /+1$ began at only position +1 during exponential phase and at both positions -1 and +1 during stationary phase. In the context of the $-1 /+1$ TA derivative, ectopic production of either Orn or $\mathrm{NrnB}$ during stationary phase eliminated transcription initiation from position -1 , while ectopic production of $\mathrm{NrnB}^{\mathrm{DHH}}$ had no effect (Fig. 5D). Thus, introduction of -1 / +1 TA into the context of a promoter that is not ordinarily targeted by nanoRNAs can cause that promoter to become sensitive to growth phase-dependent nanoRNA-mediated start site control. We conclude that $-1 /+1 \mathrm{TA}$ represents a nanoRNA response element that determines, in part, whether a promoter will be susceptible to nanoRNAmediated start site control.

\section{Discussion}

Here we establish that nanoRNA-mediated priming of transcription initiation occurs in wild-type cells of $E$. coli. In particular, we demonstrate in E. coli that nanoRNAmediated priming of transcription initiation controls transcription start site selection and gene expression in

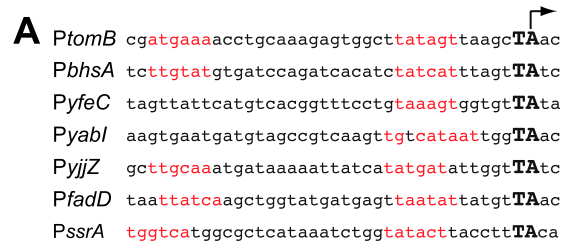

B
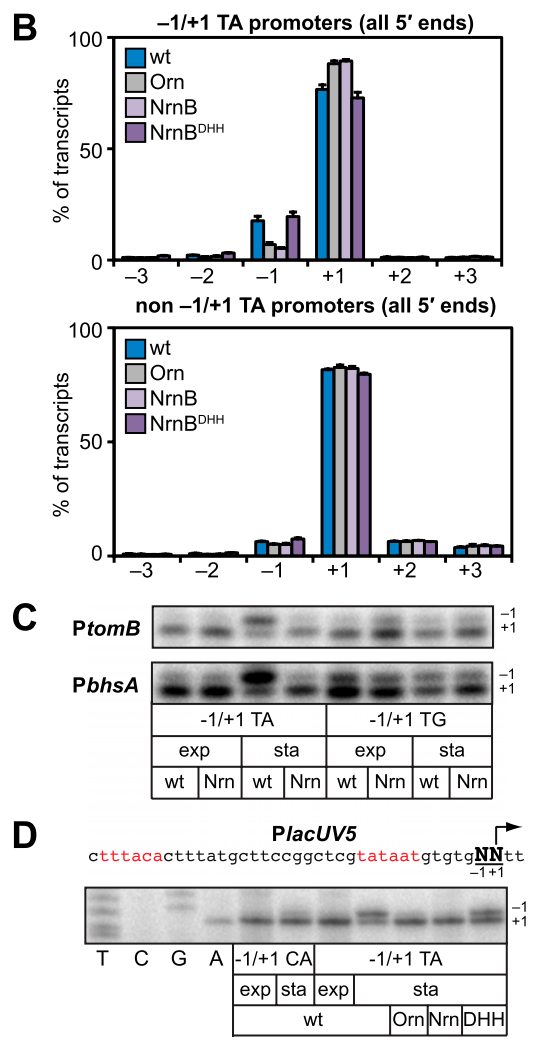

a growth phase-dependent manner. We identified seven promoters that are targeted by nanoRNA-mediated priming specifically during the stationary phase of growth. For at least five of these promoters, nanoRNA-mediated priming leads to growth phase-dependent changes in transcription start site selection. Furthermore, for at least two genes, growth phase-dependent nanoRNA-mediated priming increases the expression of these genes during stationary phase, establishing that nanoRNAs play a regulatory role.

\section{NanoRNA-mediated priming is targeted to promoters carrying a nanoRNA response element}

Our findings suggest that growth phase-dependent nanoRNA-mediated priming of transcription initiation is targeted to a specific set of promoters. In particular, each of the promoters bearing start sites that were significantly affected by ectopic production of a nanoRNase during the stationary phase of growth carried a $\mathrm{T}$ at position -1 and an $\mathrm{A}$ at position +1 (Fig. 5A). Mutating this $-1 /+1$ TA sequence element eliminated the effect of ectopic production of a nanoRNase on transcription start site selection (Fig. 5C). Furthermore, start site selection at a heterologous promoter became sensitive to ectopic production of a functional nanoRNase upon introduction of a $\mathrm{T}$ at

Figure 5. $-1 /+1$ TA represents a nanoRNA response element. (A) Sequences of seven promoters with start sites that were significantly affected by ectopic production of a nanoRNase during the stationary phase of growth. Putative -10 and -35 elements of each promoter are highlighted in red. Position +1 is indicated by the arrow. $(B)$ Average percentage of all transcripts initiated at positions -3 to +3 for the 44 promoters listed in Supplemental Table 2 that carry a $\mathrm{T}$ at position -1 and an $\mathrm{A}$ at position +1 (top) or the 181 promoters listed in Supplemental Table 2 that did not carry a $\mathrm{T}$ at position -1 and an $\mathrm{A}$ at position +1 (bottom). Analysis was performed using RNA transcripts isolated from $E$. coli cells during the stationary phase of growth that harbored an empty plasmid (wt) or a plasmid that specifies production of E. coli Orn, B. subtilis $\mathrm{NrnB}$, or $\mathrm{NrnB}^{\mathrm{DHH}}$. Plotted are the mean $+S D$ derived from two independent measurements. (C) Primer extension analysis of transcript $5^{\prime}$ ends generated during transcription initiation from the wild-type tom $B$ and $b h s A$ promoters $(-1 /+1 \mathrm{TA})$ or a derivative carrying an $A+1 G$ substitution $(-1 /+1$ TG). Analysis was performed using RNA transcripts isolated from $E$. coli cells during the exponential phase of growth (exp) or the stationary phase of growth (sta). Cells harbored a plasmid carrying the indicated promoter along with an empty plasmid (wt) or a plasmid that specifies production of $B$. subtilis NrnB. $(D)$ Primer extension analysis of transcript $5^{\prime}$ ends generated during transcription initiation from the indicated derivative of the lacUV5 promoter. Analysis was performed using RNA transcripts isolated from $E$. coli cells during the exponential phase of growth (exp) or the stationary phase of growth (sta). Cells harbored a plasmid carrying the indicated lacUV5 promoter derivative along with an empty plasmid $(\mathrm{wt})$ or a plasmid that specifies production of E. coli Orn, B. subtilis NrnB, or $\mathrm{NmB}^{\mathrm{DHH}}$. The -10 and -35 elements of the lacUV5 promoter are highlighted in red. Position +1 is indicated by the arrow. 
position -1 and an $\mathrm{A}$ at position +1 (Fig. 5D). Thus, our findings suggest that the growth phase-dependent nanoRNA-mediated priming that we observe is targeted to a specific set of promoters that carry a $-1 /+1$ TA, which we term the nanoRNA response element. In the context of these $-1 /+1$ TA promoters, nanoRNA-mediated priming produces transcripts initiating $1 \mathrm{bp}$ upstream of the primary transcription start site (i.e., from position -1 ). Such transcripts could only be generated by nanoRNAs beginning with the sequence "UA." Thus, to account for our findings that $-1 /+1$ TA promoters are preferentially targeted by growth phase-dependent nanoRNA-mediated priming, we speculate that 2- to 4-nt nanoRNAs beginning with the sequence UA preferentially accumulate during stationary phase (Fig. 6). In addition, the finding that transcripts initiating from position -1 of these promoters carry a 5' hydroxyl (Fig. 2D) suggests that the 2- to 4-nt nanoRNAs beginning with UA used to generate these transcripts also carry a 5' hydroxyl group.

We note that whereas our data suggest that $-1 /+1$ TA promoters are more likely than others to be susceptible to nanoRNA-mediated priming (Fig. 5B), not all $-1 /+1$ TA promoters appear to be targeted (Supplemental Table 2). Thus, features in addition to $-1 /+1$ TA that we have yet to identify likely influence how susceptible a given promoter is to nanoRNA-mediated priming. We further note that our findings that $-1 /+1$ TA promoters are more susceptible than other promoters to ectopic production of Orn and NrnB could, in principle, be explained on the basis that Orn and NrnB preferentially degrade nanoRNAs beginning with the sequence UA. We consider this possibility unlikely for two reasons. First, Orn and NrnB are members of two distinct protein families, decreasing the likelihood that they would share similar substrate sequence specificities. Second, biochemical characterization of the sequence specificity of Orn did not reveal any bias for the preferential degradation of nanoRNAs beginning with the sequence UA (Datta and Niyogi 1975).

\section{NanoRNA-mediated priming as a mechanism to influence gene expression}

The most extensively studied group of regulatory small RNAs in bacteria are between $50 \mathrm{nt}$ and $400 \mathrm{nt}$ in size and typically function by base-pairing with target transcripts (for review, see Waters and Storz 2009). We found that growth phase-dependent nanoRNA-mediated priming increased the expression of at least two genes, tom $B$ and bhs $A$ (Fig. 4), indicating that nanoRNAs play a regulatory role. Our findings thus establish nanoRNAs as a previously undocumented class of regulatory small RNAs that act via a previously undocumented mode of action: direct incorporation into a target transcript. In principle, there are at least three nonmutually exclusive mechanisms by which nanoRNA-mediated priming could serve to increase the abundance of the tom $B$ and $b h s A$ transcripts (for review, see Nickels and Dove 2011). First, de novo initiation from the tom $B$ promoter or the bhs $A$ promoter might be inefficient during stationary phase compared with nanoRNA-primed initiation. Thus, nanoRNA-mediated priming may simply increase the amount of transcription initiation from these promoters. Second, the addition of a $\mathrm{U}$ to the $5^{\prime}$ end of the tomB or bhs $A$ transcripts by nanoRNA-mediated priming might increase the stability of the resulting transcript through effects on RNA secondary structure. Third, the incorporation of a hydroxyl at the $5^{\prime}$ end of the tom $B$ or bhs $A$ transcripts by nanoRNA-mediated priming might increase the stability of these transcripts compared with de novo initiated tom $B$ or bhs $A$ transcripts carrying a $5^{\prime}$ triphosphate. With regard to the third mechanism, it has been shown that the endonuclease RNase E, which initiates the decay of most mRNAs in E. coli, does not work efficiently on transcripts carrying a $5^{\prime}$ hydroxyl (Tock et al. 2000; Jiang and Belasco 2004; Koslover et al. 2008). Furthermore, it has been demonstrated in vivo that transcripts carrying a 5' hydroxyl (generated through the self cleavage of a hammerhead ribozyme) can be more stable than transcripts of identical sequence carrying a $5^{\prime}$ triphosphate (Celesnik et al. 2007).

\section{NanoRNA-mediated priming occurs in a growth phase-dependent manner}

We found that nanoRNA-mediated priming was manifest during the stationary phase of growth but not during the exponential phase of growth. It is well established that NTP concentrations are reduced during stationary

\section{Exponential phase}

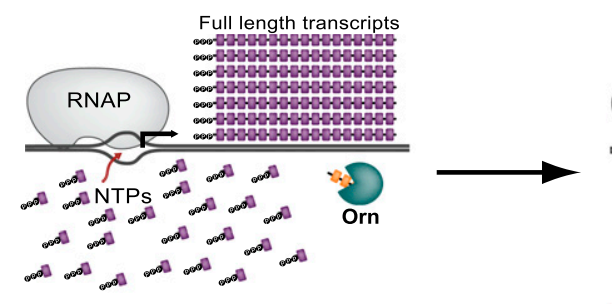

Stationary phase

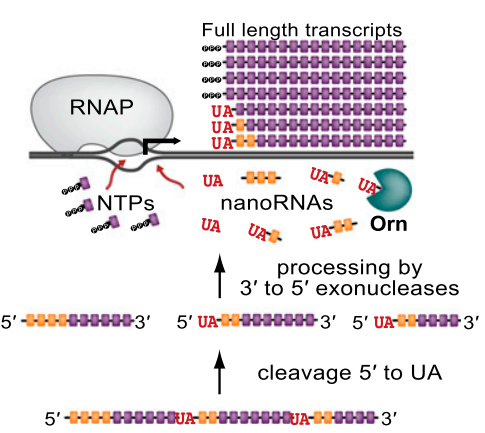

Figure 6. Model of growth phase-dependent nanoRNA-mediated priming of transcription initiation. The accumulation of nanoRNAs beginning with the sequence UA and carrying a $5^{\prime}$ hydroxyl during the stationary phase of growth is depicted. We propose that the accumulation of these nanoRNAs occurs through the degradation of full-length transcripts initiated by a cleavage event specifically targeted to the phosphodiester bond $5^{\prime}$ to the sequence UA (in red). Subsequent processing of these fragments by $3^{\prime}$-to- $5^{\prime}$ exonucleases results in the generation of 2- to 4-nt nanoRNAs that are either degraded by Orn or used to initiate transcription from promoters carrying a $-1 /+1$ TA sequence element. 
phase compared with exponential phase (Murray et al. 2003; Buckstein et al. 2008). Since nanoRNAs will compete with NTPs for use by RNA polymerase during transcription initiation, the reduction in NTP concentrations that accompanies the transition from exponential to stationary phase may contribute to the observed growth phase dependence of nanoRNA-mediated priming. In principle, alterations in the metabolism of nanoRNAs during the stationary phase of growth might also contribute to the extent to which nanoRNA-mediated priming occurs. However, Western blot analysis indicated that the concentration of Orn does not decrease during stationary phase relative to exponential phase (Supplemental Fig. 1). Thus, a growth phase-dependent reduction in the metabolism of nanoRNAs likely does not explain why nanoRNA-mediated priming is manifest during stationary phase.

As mentioned above, our findings suggest that 2- to 4-nt UA nanoRNAs carrying a 5' hydroxyl are responsible for the observed growth phase-dependent nanoRNA-mediated priming. To account for our observations, we propose that 2- to 4-nt UA nanoRNAs carrying a $5^{\prime}$ hydroxyl preferentially accumulate during the stationary phase of growth. We posit that such nanoRNAs are generated by the decay of full-length RNA transcripts through a multistep process that is initiated by a cleavage event specifically targeted to the phosphodiester bond $5^{\prime}$ to the sequence UA, which generates a 5 ' hydroxyl end (Fig. 6). Subsequent processing of these fragments by the major 3'-to-5' exonucleases in E. coli (polynucleotide phosphorylase, RNase II, or RNase R) would leave terminal 2- to 4-nt UA nanoRNAs carrying a 5' hydroxyl that could be either further degraded by Orn or used to prime transcription initiation.

In summary, our findings identify nanoRNAs as a previously undocumented class of regulatory small RNAs that can influence both transcription start site selection and gene expression. Current objectives are to define the mechanism by which nanoRNA-mediated priming of transcription initiation influences gene expression, establish why nanoRNA-mediated priming occurs in a growth phase-dependent manner, and further define why certain promoters are targeted by nanoRNA-mediated priming while others are not. In addition, the approaches that we developed here to identify nanoRNA-mediated priming in E. coli can, in principle, be used to define the extent to which nanoRNA-mediated priming of transcription initiation occurs in other organisms.

\section{Materials and methods}

Experiments were performed in LB medium (Miller formulation, EMD Chemicals) using E. coli strain MG1655. The following antibiotics and inducers were used: $25 \mu \mathrm{g} / \mathrm{mL}$ chloramphenicol, $10 \mu \mathrm{g} / \mathrm{mL}$ gentamicin, and $1 \mathrm{mM}$ IPTG. Cells were grown overnight at $37^{\circ} \mathrm{C}$ without IPTG, back-diluted to an $\mathrm{OD}_{600}$ of $\sim 0.035$ into flasks containing medium with IPTG, and grown at $37^{\circ} \mathrm{C}$. Exponential phase samples were harvested at an $\mathrm{OD}_{600}$ of $\sim 0.5$. Stationary phase samples were harvested after $23 \mathrm{~h}$ of growth $\left(\mathrm{OD}_{600} \sim 3.5\right)$. RNA was isolated from cells as described (Goldman et al. 2011). Ectopic production of Orn, NrnB, and
$\mathrm{NrnB}^{\mathrm{DHH}}$ was confirmed by Western blot analysis (Supplemental Fig. 1).

\section{Primer extension}

RNA was isolated from cells containing a plasmid carrying the indicated promoter and a plasmid directing the synthesis of the indicated nanoRNase (see the Supplemental Material for a detailed description of the plasmids). Primer extension was done as described (Goldman et al. 2011) using a radiolabeled probe (5'ATTTTGCAGACCTCTCTGCC-3') complementary to sequences present only on the plasmid vector.

\section{Quantitative real-time RT-PCR}

cDNA synthesis was performed as described (Wolfgang et al. 2003). The abundance of bhsA transcripts relative to rpoD transcripts was determined by quantitative real-time RT-PCR using the iTaq SYBR Green kit (Bio-Rad) and an ABI Step One Plus instrument (Applied Biosystems). Experiments were performed at least three times on separate occasions, and a representative data set is shown. The following primers were used: bhsA-RTF (GCTCCATGTCATTTGCCAGCTTTG), bhsA-RTR (GCGTTAGCACTGATTGTACCGACT), rpoD-RTF (ATGTAC ATGCGTGAAATGGGCACC), and rpoD-RTR (TCCGGATATT CAGCAACGGAGCAT).

\section{High-throughput sequencing}

The procedure used to identify transcription start sites and quantify transcripts initiating at a particular position by highthroughput sequencing using an Applied Biosystems SOLiD system (version 4.0) was essentially as described in Goldman et al. (2011). The following modification of the protocol was made to analyze $5^{\prime}$ monophosphate ends: rRNA-depleted RNAs were ligated directly to the $5^{\prime}$ SOLiD adaptor. The following modifications of the protocol were made to analyze $5^{\prime}$ hydroxyl ends: rRNA-depleted RNAs were treated with RNA $5^{\prime}$ polyphosphatase (to convert $5^{\prime}$ triphosphate ends to $5^{\prime}$ monophosphate ends), followed by Terminator $5^{\prime}$-Phosphate-Dependent Exonuclease (to remove transcripts with $5^{\prime}$ monophosphate ends), followed by OptiKinase (Affymetrix) treatment (to covert $5^{\prime}$ hydroxyl ends to $5^{\prime}$ monophosphate ends suitable for ligation to the $5^{\prime}$ SOLiD adaptor). The resultant 5' monophosphatecarrying transcripts were then ligated to the $5^{\prime} \mathrm{SOLiD}$ adaptor.

Reads that mapped uniquely with zero mismatches to a single position in the E. coli MG1655 genome were identified using Bioscope 1.3 (Applied Biosystems). Identification of transcription start sites $(+1)$ and calculations of the percentage of transcripts initiated between -3 and +3 were as described (Goldman et al. 2011). Graphs were generated using the values provided in Supplemental Table 6.

Start sites that were affected by ectopic production of either Orn or NrnB in a manner consistent with a reduction of nanoRNAmediated priming met the following criteria in the analysis of each biological replicate. Ectopic production of Orn alone and $\mathrm{NrnB}$ alone caused $25 \%$ fewer of the total transcripts to be initiated from positions $-3,-2$, or -1 compared with cells containing wild-type concentrations of nanoRNAs or cells in which $\mathrm{NrnB}^{\mathrm{DHH}}$ was ectopically produced. None of the 270 start sites analyzed during exponential phase met these criteria (Supplemental Table 1), whereas seven of the 225 start sites analyzed during stationary phase did (Supplemental Tables 2, 3).

\section{Plasmids}

Plasmid pPSV38, which confers resistance to gentamicin, was used as an empty vector control in the experiments in which 
a nanoRNase was ectopically produced. Plasmid pEcOrn confers resistance to gentamicin and expresses the orn gene from E. coli strain MG1655 under the control of an IPTG-inducible lacUV5 promoter flanked by two lac operators. Plasmid pNrnB-VSVG confers resistance to gentamicin and expresses the $n r n B$ gene from B. subtilis strain PY73 fused to a VSV-G epitope tag under the control of an IPTG-inducible lacUV5 promoter flanked by two lac operators. Plasmid $\mathrm{pNrnB}^{\mathrm{DHH}}-\mathrm{VSVG}$ is the same as pNrnB-VSVG, with the exception that the $n r n B$ gene carries three amino acid substitutions (D86A, H87A, and H88A) that were introduced by site-directed mutagenesis into pNrnB-VSVG.

Plasmid pBEN 504 carries sequences extending from -100 to +15 of the promoter associated with $t o m B$ (the genomic position of +1 in the annotated MG1655 genome is 480,019 ) fused to the $\mathrm{tR}^{\prime}$ terminator cloned into the HindIII and SalI sites of plasmid pACYC184 (New England Biolabs). Plasmid pBEN 556 is identical to pBEN 504, with the exception that it carries an A+1G substitution of the promoter associated with tomB. Plasmid pBEN 516 carries sequences extending from -100 to +15 of the promoter associated with bhs $A$ (the genomic position of +1 in the annotated MG1655 genome is $1,168,242)$ fused to the $\mathrm{tR}^{\prime}$ terminator cloned into the HindIII and SalI sites of plasmid pACYC184. Plasmid pBEN 562 is identical to pBEN 516, with the exception that it carries an $\mathrm{A}+1 \mathrm{G}$ substitution of the promoter associated with bhsA. Plasmid pBEN 493 carries a lacUV5 promoter derivative carrying a TA at position $-1 /+1$ fused to the $\mathrm{tR}^{\prime}$ terminator cloned into the HindIII and SalI sites of plasmid pACYC184. Plasmid pBEN 495 carries a lacUV5 promoter derivative carrying a " $\mathrm{CA}^{\text {" }}$ at position $-1 /+1$ fused to the $\mathrm{tR}^{\prime}$ terminator cloned into the HindIII and SalI sites of plasmid pACYC184.

A detailed description of the construction of each plasmid is provided in the Supplemental Material.

\section{Northern blot analysis}

Ten micrograms of total RNA was mixed with an equal volume of glyoxal sample loading dye (Ambion) and heated for $30 \mathrm{~min}$ at $50^{\circ} \mathrm{C}$ before being electrophoresed on a $1 \%$ agarose gel and subsequently transferred to a positively charged nylon membrane (Roche), essentially as described in the NorthernMax-Gly kit (Ambion). Digoxigenin (DIG)-labeled riboprobes complementary to the bhs $A$ and tom $B$ transcripts were generated using $\mathrm{T} 7$ RNA polymerase according to the DIG RNA labeling kit (SP6) T7) (Roche). DIG-labeled riboprobes were detected using the CDP-Star kit (Roche). Experiments were performed four independent times with similar results. Representative data are presented in Figure 4A. A DIG labeled riboprobe specific for tom $B$ detected two prominent transcripts that were sensitive to ectopic production of $\mathrm{NrnB}$ in stationary phase cells. The larger of these transcripts migrated between the 575-nt and 1049-nt fragments of a DIG-labeled RNA molecular weight marker (Roche), whereas the smaller transcript migrated between the 483-nt and 575-nt fragments of the marker. A DIG-labeled riboprobe specific for $b h s A$ detected a single transcript that was sensitive to ectopic production of $\mathrm{NrnB}$ in stationary phase cells. This transcript migrated between the 310-nt and 483-nt fragments of the marker.

\section{Acknowledgments}

This work was supported by an NRSA fellowship (S.R.G.); NIH grants AI076608 (J.L.), GM096454 (B.E.N. and S.L.D.), and GM088343 (B.E.N.); and a Pew Scholars Award (B.E.N.). We thank A. Hochschild for discussion.

\section{References}

Arditti RR, Scaife JG, Beckwith JR. 1968. The nature of mutants in the lac promoter region. J Mol Biol 38: 421-426.

Buckstein MH, He J, Rubin H. 2008. Characterization of nucleotide pools as a function of physiological state in Escherichia coli. J Bacteriol 190: 718-726.

Celesnik H, Deana A, Belasco JG. 2007. Initiation of RNA decay in Escherichia coli by $5^{\prime}$ pyrophosphate removal. Mol Cell 27: 79-90.

Datta AK, Niyogi K. 1975. A novel oligoribonuclease of Escherichia coli. II. Mechanism of action. J Biol Chem 250: 73137319.

Fang M, Zeisberg WM, Condon C, Ogryzko V, Danchin A, Mechold U. 2009. Degradation of nanoRNA is performed by multiple redundant RNases in Bacillus subtilis. Nucleic Acids Res 37: 5114-5125.

García-Contreras R, Zhang XS, Kim Y, Wood TK. 2008. Protein translation and cell death: The role of rare tRNAs in biofilm formation and in activating dormant phage killer genes. PLOS ONE 3: e2394. doi: 10.1371/journal.pone.0002394.

Ghosh S, Deutscher MP. 1999. Oligoribonuclease is an essential component of the mRNA decay pathway. Proc Natl Acad Sci 96: $4372-4377$.

Goldman SR, Sharp JS, Vvedenskaya IO, Livny J, Dove SL, Nickels BE. 2011. NanoRNAs prime transcription initiation in vivo. Mol Cell 42: 817-825.

Grachev M, Zaychikov E, Ivanova E, Komarova N, Kutyavin I, Sidelnikova N, Frolova I. 1984. Oligonudeotides complementary to a promoter over the region $-8 \ldots+2$ as transcription primers for E. coli RNA polymerase. Nucleic Acids Res 12: 8509-8524.

Jiang X, Belasco JG. 2004. Catalytic activation of multimeric RNase E and RNase G by 5'-monophosphorylated RNA. Proc Natl Acad Sci 101: 9211-9216.

Koslover DJ, Callaghan AJ, Marcaida MJ, Garman EF, Martick M, Scott WG, Luisi BF. 2008. The crystal structure of the Escherichia coli RNase E apoprotein and a mechanism for RNA degradation. Structure 16: 1238-1244.

Liu C, Heath LS, Turnbough CL Jr. 1994. Regulation of pyrBI operon expression in Escherichia coli by UTP-sensitive reiterative RNA synthesis during transcriptional initiation. Genes Dev 8: 2904-2912.

Liu MF, Cescau S, Mechold U, Wang J, Cohen D, Danchin A, Boulouis HI, Biville F. 2012. Identification of a new nanoRNase in Bartonella. Microbiology 158: 886-895.

Livak KJ, Schmittgen TD. 2001. Analysis of relative gene expression data using real-time quantitative PCR and the $2^{-\Delta \Delta \mathrm{C}(\mathrm{T})}$ method. Methods 25: 402-408.

Mechold U, Fang G, Ngo S, Ogryzko V, Danchin A. 2007. YtqI from Bacillus subtilis has both oligoribonuclease and pApphosphatase activity. Nucleic Acids Res 35: 4552-4561.

Meng Q, Turnbough CL Jr, Switzer RL. 2004. Attenuation control of pyrG expression in Bacillus subtilis is mediated by CTP-sensitive reiterative transcription. Proc Natl Acad Sci 101: 10943-10948.

Mermod M, Magnani D, Solioz M, Stoyanov JV. 2012. The copper-inducible ComR (YcfQ) repressor regulates expression of ComC (YcfR), which affects copper permeability of the outer membrane of Escherichia coli. Biometals 25: 33-43.

Murray HD, Schneider DA, Gourse RL. 2003. Control of rRNA expression by small molecules is dynamic and nonredundant. Mol Cell 12: 125-134.

Nickels BE, Dove SL. 2011. NanoRNAs: A class of small RNAs that can prime transcription initiation in bacteria. J Mol Biol 412: $772-781$. 
Qi F, Turnbough CL Jr. 1995. Regulation of $\operatorname{codBA}$ operon expression in Escherichia coli by UTP-dependent reiterative transcription and UTP-sensitive transcriptional start site switching. J Mol Biol 254: 552-565.

Ruetsch N, Dennis D. 1987. RNA polymerase. Limit cognate primer for initiation and stable ternary complex formation. I Biol Chem 262: 1674-1679.

Tock MR, Walsh AP, Carroll G, McDowall KJ. 2000. The CafA protein required for the $5^{\prime}$-maturation of $16 \mathrm{~S}$ rRNA is a $5^{\prime}$-end-dependent ribonuclease that has context-dependent broad sequence specificity. J Biol Chem 275: 8726-8732.

Tu AH, Turnbough CL Jr. 1997. Regulation of upp expression in Escherichia coli by UTP-sensitive selection of transcriptional start sites coupled with UTP-dependent reiterative transcription. I Bacteriol 179: 6665-6673.

Waters LS, Storz G. 2009. Regulatory RNAs in bacteria. Cell 136: 615-628.

Wolfgang MC, Lee VT, Gilmore ME, Lory S. 2003. Coordinate regulation of bacterial virulence genes by a novel adenylate cyclase-dependent signaling pathway. Dev Cell 4: 253-263.

Zhang XS, Garcia-Contreras R, Wood TK. 2007. YcfR (BhsA) influences Escherichia coli biofilm formation through stress response and surface hydrophobicity. J Bacteriol 189: 30513062 . 


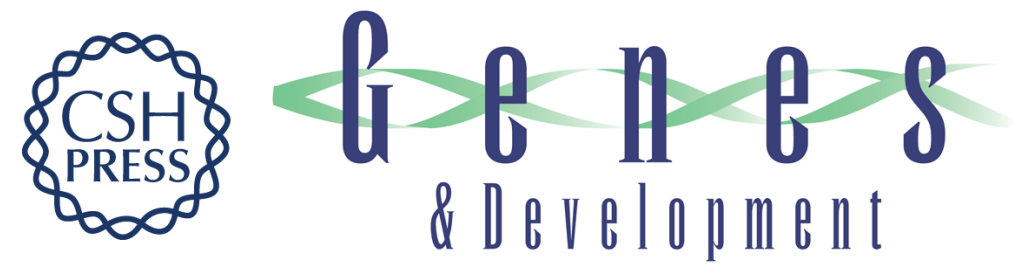

\section{Growth phase-dependent control of transcription start site selection and gene expression by nanoRNAs}

Irina O. Vvedenskaya, Josh S. Sharp, Seth R. Goldman, et al.

Genes Dev. 2012, 26:

Access the most recent version at doi:10.1101/gad.192732.112

Supplemental http://genesdev.cshlp.org/content/suppl/2012/07/02/26.13.1498.DC1
Material

References This article cites 26 articles, 10 of which can be accessed free at:

http://genesdev.cshlp.org/content/26/13/1498.full.html\#ref-list-1

License

Email Alerting

Receive free email alerts when new articles cite this article - sign up in the box at the top

Service

right corner of the article or click here.

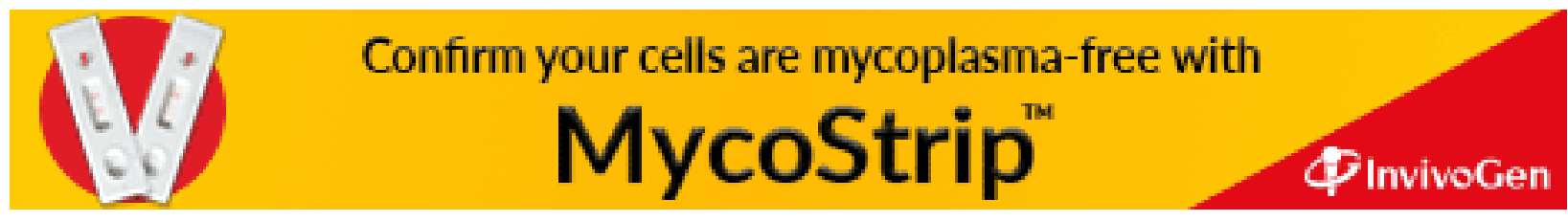

